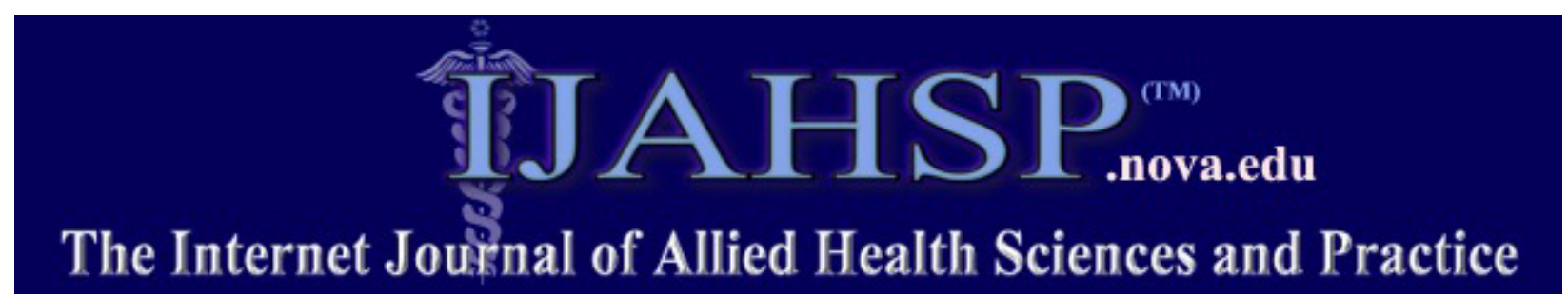

A Peer Reviewed Publication of the College of Health Care Sciences at Nova Southeastern University

Dedicated to allied health professional practice and education

http://ijahsp.nova.edu Vol. 10 No. 3 ISSN 1540-580X

\title{
Neuromuscular Electrical Stimulation in the Early Intervention Population: A Series of Five Case Studies
}

\author{
Kelly L. Rice, OTR/L \\ Occupational Therapist, Sovia Therapy, Mechanicsburg, Pennsylvania
}

United States

CITATION: Rice K. Neuromuscular Electrical Stimulation in the Early Intervention Population: A Series of Five Case Studies. The Internet Journal of Allied Health Sciences and Practice. July 2012. Volume 10 Number 3.

\begin{abstract}
The use of Neuromuscular Electrical Stimulation (NMES) to improve swallowing function in the Early Intervention home-based population with pharyngeal phase dysphagia is described using a case series design. Five children had Modified Barium Swallow studies (MBSS) completed followed by a course of swallowing therapy. The children had NMES administered home-based for 60-minute sessions. The number of treatment sessions ranged from 11 to 63 , and the MBSS was repeated at the end of therapy. All five children improved functional swallowing as documented by the attending radiologist's reports. This case series design provides data that may support the use of NMES in the Early Intervention population as a treatment modality for pharyngeal phase dysphagia.
\end{abstract}

\section{INTRODUCTION}

Infants and toddlers in the Early Intervention System have unique requirements in regards to therapeutic intervention. The purpose of Early Intervention (EI) is to "provide supports and services to infants/toddlers and young children with disabilities and their caregivers so that they may help the child grow and develop."1 Therapeutic services are typically provided weekly or on a consultative basis in the child's natural environment and while the child is participating in daily routines (e.g. eating by mouth, bathing, assisting with dressing skills). Children with feeding and swallowing issues as defined by the Pennsylvania Standards for Early Childhood are seen by an occupational therapist (OTR) or speech language pathologist (SLP) during a meal or snack time. ${ }^{2}$ Strategies pertaining to improving the child's developmental performance are discussed with caregivers, and suggestions are provided about approaches, interventions, and strategies that might improve feeding and swallowing and might enhance the child's overall development. Criteria defined in the Pennsylvania Standards include self feeding with fingers and utensils as well as drinking from bottles and cups. Avoiding choking instances by presenting foods in an age appropriate method is also outlined as appropriate scope of practice in El. Infants and children who demonstrate an inability to manage foods safely may have a deficit in either the oral or pharyngeal phase of their swallowing function. Oral phase dysphagia would include an inability to properly form a cohesive bolus in the mouth or inability to organize the food in the mouth for swallowing. Pharyngeal phase dysphagia may include residue in the structures in the pharynx and may include aspiration into the lungs of foods or liquids.

The MBSS is often used as a tool to diagnose an oral or pharyngeal phase dysphagia. According to Logemann, the MBSS is used to "(a) examine the anatomy and physiology of the oral cavity and pharynx during deglutition, (b) identify the disorders in movement patterns of orophayrngeal structures that control the bolus and cause aspiration or inefficient swallowing (residue), and (c) define treatment strategies that will eliminate aspiration and/or increase swallow efficiency." 3 Infants and toddlers are positioned for the study upright in a feeder seat or portable highchair as they are fed in their home environment. In most instances, the person conducting the feeding during a MBSS is a parent. Foods are covered in liquid barium or barium paste, and then given to the child to eat or drink. Radiation is emitted and the tissues and bony areas of the child's face and neck can

(C) The Internet Journal of Allied Health Sciences and Practice, 2012 
be seen on x-ray. Barium coated foods are accented and the exact path of the swallow can be followed. Reporting of the MBSS is dependent on the facility that conducts the study. Each radiologist describes the outcome of the study in a narrative report generally commenting on strength of swallowing musculature as well as evidence of penetration or aspiration. The attending SLP will also generate a report about the study outcomes in either a narrative or checklist format. Typically, a description of the study environment is given, and how the child responded to presentation of various food and liquid consistencies is reported.

NMES is the use of electrical stimulation for activation of muscles through stimulation of the intact peripheral motor nerves. The major treatment goals are to strengthen weak muscles and to help in the recovery of motor control.4,11 The Vital Stim® Therapy Unit is a dual-channel, electrically isolated device with miniature safety connectors. The unit is biphasic with a zero net $D C$ and can deliver up to 100 volts maximum with no load. The dual intensity has a 0 to $25 \mathrm{~mA}$ peak current output with adjustable current. The pulse rate of the device is fixed at $80 \mathrm{~Hz}$ and the pulse duration is also fixed at $300 \mu$ seconds under normal operation. ${ }^{5}$ (see Picture 1) Vital Stim ${ }^{8}$ therapy is a FDA cleared device to promote swallowing through the application of NMES to the swallowing musculature. Four hundred forty-six (446) adult stroke patients demonstrated safe and effective treatment outcomes as a result of NMES and provided the basis for FDA approval of the device and electrodes. ${ }^{6}$ The FDA imposed restrictions on the device so that it would not be used in any other way than that intended for use in strengthening musculature for swallowing. The goal of NMES is to strengthen muscles to improve motor control of the swallowing mechanism. ${ }^{4}$

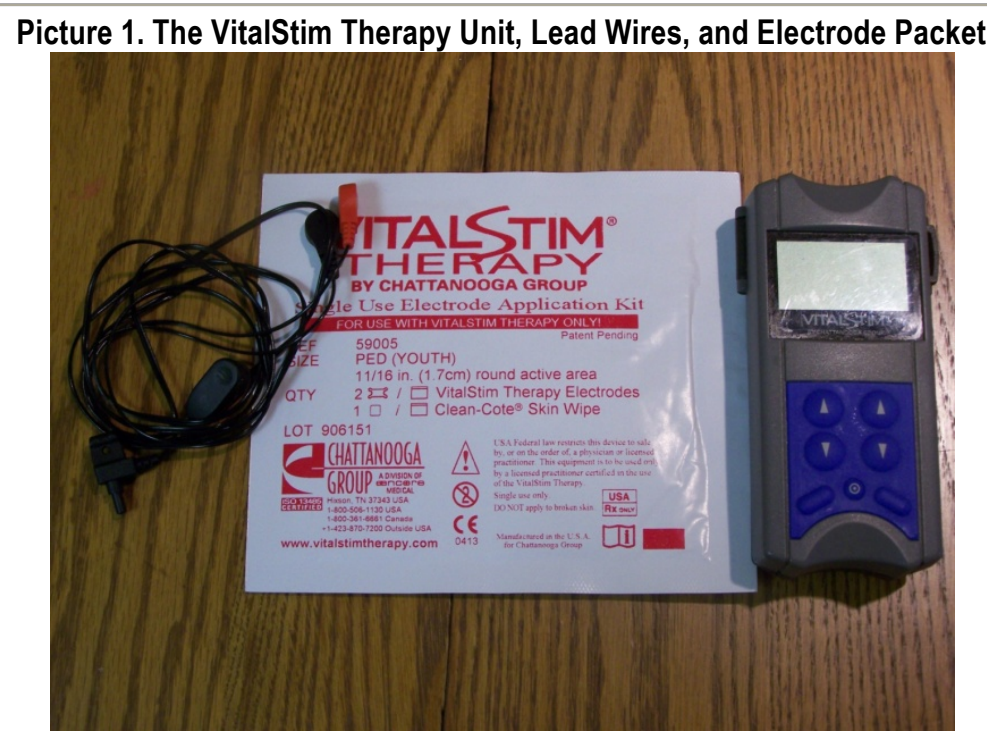

The use of NMES in the treatment of swallowing disorders in the adult population has been documented in the literature. Blumenfeld reported that patients $(n=40)$ who received Electrical Stimulation (ES) had evidence of fewer treatments and required shorter hospitalizations than those patients who had thermal stimulation. Swallow scores also improved for the ES group as compared to the group that received thermal stimulation. ${ }^{7}$ Carnaby-Mann and Crary described the effectiveness of ES with concurrent standardized exercise regimens on swallowing function in chronic dypshagia patients. They found that $80 \%$ of the patients treated demonstrated significant improvement in clinical swallowing ability, functional oral intake, weight gain, and patient perception of swallowing ability. ${ }^{8}$ Xia et al. concluded a study comparing post-stroke patients in three groups, (conventional swallowing therapy, VitalStim ${ }^{\circledR}$ therapy only, and VitalStim ${ }^{\circledR}$ combined with conventional therapy). Their results indicated that the patients who received both VitalStim $®$ and conventional therapy scored higher than patients who received just conventional swallowing therapy or just VitalStim ${ }^{\circledR} .{ }^{12}$ Xia et al. went on to conclude that the VitalStim ${ }^{\circledR}$ therapy coupled with conventional swallowing therapy is the best alternative for improving post-stroke dysphagia. ${ }^{12}$ Ryu et al. conducted a study on the effects of ES on dysphagia following treatment for head and neck cancer. They concluded that patients with dysphagia caused by treatment for head and neck cancers showed greater improvements on the Functional Dysphagia Scale (FDS) following NMES combined with conventional rehabilitation treatment than following conventional rehabilitation treatment alone. ${ }^{13}$ Oh et al. documented the average pharyngeal transit time (PTT) in young adults was lower than in healthy older adults. FDS of the oral phase was larger in the older adults than in the young adults. After NMES for 2 weeks in older adults, there was a significant improvement in the averages for PTT and FDS. ${ }^{14}$ Langdon and Blacker outlined in their review article a call for more research of NMES so that therapists would know when to apply the modality and when to use conventional therapies. ${ }^{15}$ Barikroo and Lam documented a case report comparing the effects of conventional swallowing therapy compared to using NMES in a 
patient with encephalitis. The patient in their study (age 49) demonstrated significant improvement in clinical and functional swallowing ability as well as laryngeal elevation after intervention. ${ }^{16}$

Pediatric research in the use of this modality is limited, however, and protocols for effective intervention in this population are not well defined. Christiaanse et al. described a consecutive sample of 30 pediatric patients where NMES was used after conventional therapy had failed. Patients had multiple etiologies: congenital anomaly $(n=17)$, acquired CNS lesion $(n=7)$, or unknown etiology $(n=6)$. Patients received electrical stimulation to the anterior neck for an average of 22 sessions of one hour each, until the swallow had improved or until no further improvement was noted. ${ }^{9}$ Outcome measures were a swallow score on a non-validated scale and a videofluoroscopy evaluation. The authors reported that 17 out of 30 patients improved, with 5 out of 30 achieving a normal swallow.

The purpose of this case series is to describe the use of a home-based pediatric treatment protocol which includes the use of NMES on five children with pharyngeal phase dysphagia. Children were identified as qualifying for El services by demonstrating a delay of $25 \%$ or greater in one or more areas of development with a specific need for assistance in eating or swallowing. A referral was made by the child's independent evaluation team or Individualized Family Service Plan team for improvement of feeding skills, and appropriate prescriptions were obtained from the child's physician.

\section{SUBJECTS}

Five children were seen at home through the Early Intervention System one to two times per week after a diagnosed pharyngeal phase dysphagia by MBSS. There were three boys and two girls in the sample, with an age range of 3 months to 32 months (median age 17.4 months) at the start of treatment. Informed consent was obtained from each parent to participate in this observational case series design and appropriate prescriptions were obtained from the child's physician.

Table 1. Client Background Information

\begin{tabular}{|l|l|l|l|l|}
\hline Name & Age & Gender & Diagnosis & No. of NMES sessions \\
\hline Client \#1 & $\begin{array}{l}14 \\
\text { months }\end{array}$ & Male & $\begin{array}{l}\text { Developmental Delay, ruling out } \\
\text { genetic defect }\end{array}$ & 17 \\
\hline Client \#2 & 3 months & Male & $\begin{array}{l}\text { Perinatal asphyxia requiring 15 } \\
\text { minute resuscitation, small } \\
\text { ventricular hemorrhage }\end{array}$ & 11 \\
\hline Client \#3 & $\begin{array}{l}29 \\
\text { months }\end{array}$ & Male & $\begin{array}{l}\text { Born at 26 weeks, 4 days } \\
\text { gestational age, bronchopulmonary } \\
\text { dysplasia }\end{array}$ & 41 \\
\hline Client \#4 & $\begin{array}{l}32 \\
\text { months }\end{array}$ & Female & $\begin{array}{l}\text { All organs are reversed, extra half } \\
\text { vertebrae, missing her spleen }\end{array}$ & 63 \\
\hline Client \#5 & 9 months & Female & $\begin{array}{l}\text { Failure to thrive, g- and j-tube } \\
\text { placement, Nissen fundoplication }\end{array}$ & 26 \\
\hline
\end{tabular}

\section{METHODS}

In this case series design, each child had a MBSS prior to intervention and a second follow up study conducted at the end of therapy. For the purposes of this case series, the radiologist data indicated in the reports were used to compare pre- and postNMES findings. The SLP that was also present for the study completed reports that ranged from checklists to extensive narratives. At times, the SLP's MBSS report conflicted with the radiologist's findings and in other circumstances, was not available for inclusion in this series even when requested from the hospital that conducted the study. NMES was administered by the same certified occupational therapist to the child at home one to two times per week utilizing the VitalStim ${ }^{\circledR}$ unit for one hour sessions. An occupational therapist administered NMES because in this geographical region in El, occupational therapists generally see children with feeding and swallowing disorders. In all cases, the clients had a two-electrode placement due to small neck size, with placement horizontal above the thyroid notch (see Picture 2, Pediatric Placement A/placement 1) or horizontal around the thyroid notch (see Picture 2, Pediatric Placement B/placement 2). ${ }^{4}$ Pediatric Placement A is indicated for children that have decreased tongue base retraction, dysfunctional laryngeal excursion, and/or pooling in the valleculae. ${ }^{4}$ The electrodes stimulate extrinsic and some intrinsic tongue musculature as well as suprahyoid muscles that promote laryngeal elevation. ${ }^{4}$ Pediatric Placement $B$ is indicated for most largyneal and pharyngeal motor deficits, and the rationale behind placement in this location would be to focus on infrayhoid muscles and with sufficient intensity, intrinsic laryngeal, and pharyngeal muscles. ${ }^{4}$ 
All of the children in this series needed to improve laryngeal elevation and pharyngeal contraction to improve their swallow safety, and in some cases, children needed to improve tongue base retraction and decrease valleculae pooling. The intensity $(0$ to $25 \mathrm{~mA}$ ) was adjusted for each child to their highest tolerated level as evidenced by visible muscle contraction or the child grabbing at the electrodes. Children were given carryover programs (e.g. tucked position to drink, z-vibe to improve mouth awareness) and suggestions (e.g. pacing while eating and drinking) to complete between sessions by their parents and/or caregivers. Parents were asked to continue to provide the appropriate diet consistency (i.e. thickened liquids, thicker purees) as determined by the child's initial MBSS. In addition, oral motor stimulation techniques were demonstrated with the child, and parents were asked to continue those activities between therapy sessions. The use of z-vibe, Nuk brushes, and flavored chewy tubes were used depending on the child's need and given to caregivers to use between therapy sessions (see Picture 3). Decisions to repeat the MBSS was made when the child appeared to swallow safer using clinical observations or if ordered by the physician. According to this author, clinical signs of aspiration include wet quality to vocalizations, excessive coughing while eating, and/or parents reporting "choking" episodes. Consequently, clients appeared to be safer while swallowing if they no longer demonstrated signs of aspiration.

\section{Picture 2. Electrode Placements}

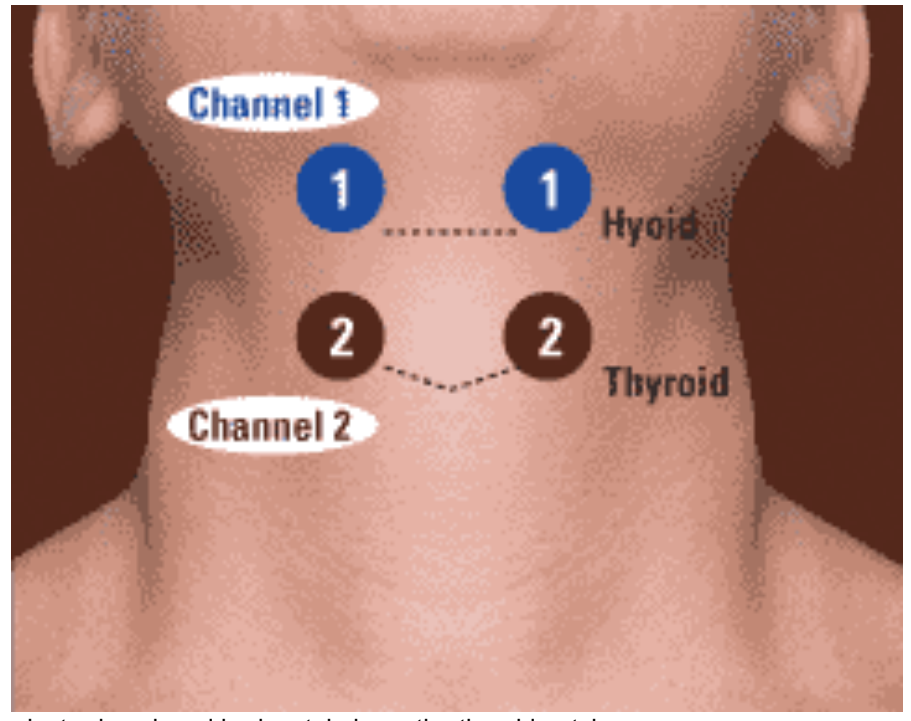

$1=$ Pediatric Placement $A-$ two electrodes placed horizontal above the thyroid notch 2= Pediatric Placement $B$ - two electrodes placed horizontal around the thyroid notch Used with permission from Vital Stim ® User Manual. Chattanooga Group, Encore Medical Corporation, Austin, TX. 2005.

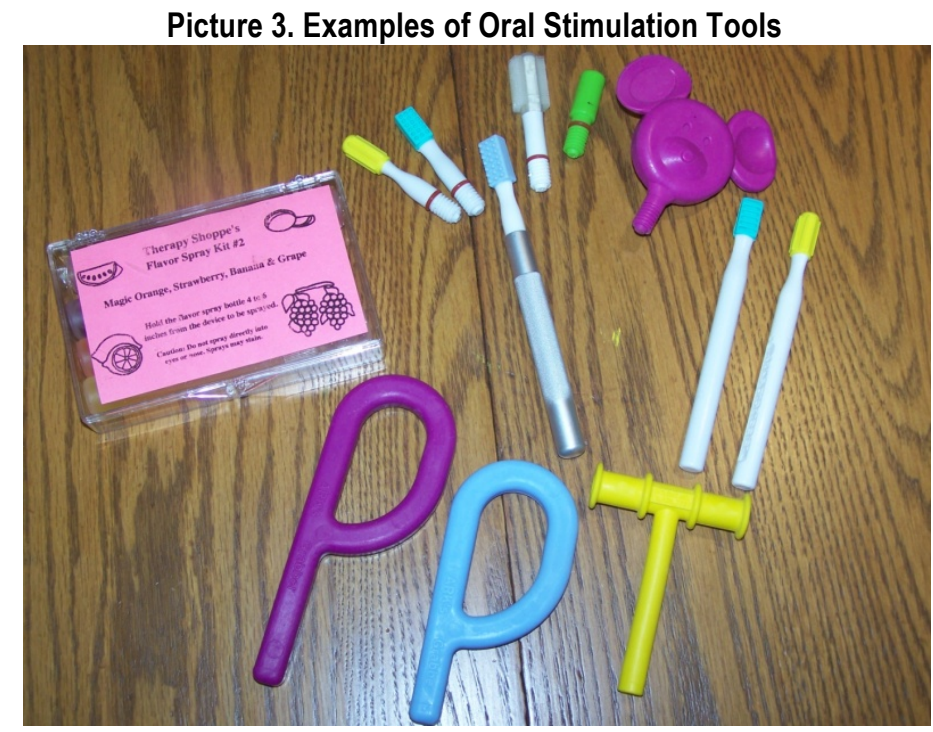


Each week, all parents reported verbally at the beginning of the therapy session to the therapist how each of the children performed while eating or completing oral motor exercises during the previous week (e.g. following vibration in their child's mouth, making funny faces in a mirror). At times, NMES was unable to be delivered due to child illness or therapist scheduling issues. Each child tolerated the stimulation by allowing the electrodes to remain in place for a full 60-minute session each time. In the pediatric El population, determining therapeutic intensity can be challenging, as the child is not generally able to describe how the stimulation feels. Evidence of a therapeutic intensity level (e.g. "grabbing" sensation) having been reached needs to be determined by the therapist administering the stimulation by assessing the child's non-verbal cues and body language. Some children indicated that the intensity was appropriate by wanting to swallow and eat more. Others had a noticeable change in the audible quality of the swallow or voice production during times when the child would babble or vocalize. Voice quality was heard while the child was eating and playing and with cervical auscultation using a stethoscope on the child's neck.

\section{RESULTS}

Client \#1 had an initial MBSS conducted, and the radiologist documented persistent silent micro aspiration with both rice cereal and thin barium by bottle. Client \#1 had 17 NMES sessions completed with a two-electrode placement just at the area of the thyroid notch (see Picture 2 - placement 2/Pediatric Placement B). This placement was chosen because the client needed general strengthening in the area of the larynx and pharynx and had general motor deficits. The client was extremely orally defensive as he had not eaten or had stimulation in his mouth since birth. Small tastes of foods were able to be given at home and eventually he would allow Nuk brush stimulation in and around his oral cavity. His tongue remained very sensitive with a hyperactive gag. Prior to his repeated MBSS, Client \#1 was demonstrating the ability to vomit small amounts of foods, so he was scheduled for a MBSS as his attending physician was concerned that he might be aspirating his vomit. The repeated MBSS was completed, and the radiologist concluded Client \#1 was safe on all consistencies such as thin liquids, purees, and bottle drinking.

Client \#2 had an initial MBSS, and the attending radiologist commented that he had a mildly compromised oral phase (e.g. some bolus formation, but not complete) with more significant compromise of the pharyngeal phase (e.g. aspiration noted). He silently aspirated on water consistency liquids (referred to as thin liquids) and had incomplete laryngeal closure. Following this study, Client \#2 had NMES sessions conducted at home for a total of 11 sessions with electrode placement at the level of the thyroid notch (see Picture 2 - placement 2/Pediatric Placement B). Liquids were thickened to at least honey consistency with the nipples on his bottles cross-hatched so that the fluid was able to be extracted. At that time, Client \#2 was only drinking with bottles, but he did allow a Nuk pacifier in his mouth occasionally to stimulate non-nutritive sucking. He was readily receptive and accepted other stimulation in his mouth like Nuk brushes and vibration in the form of a z-vibe stimulator. Client \#2 had his MBSS repeated, and was found to have a coordinated oral and pharyngeal phase as well as no aspiration or penetration with any consistency and complete laryngeal closure.

Client \#3 had an initial MBSS conducted, and the radiologist commented that he had persistent, silent aspiration on thin liquids. Following this study, Client \#3 had 41 NMES sessions conducted at his home one to two times per week with placement at the level of the thyroid notch or slightly above the thyroid notch (see Picture 2 - placement 1 and 2/Pediatric Placement A and B). All liquids were thickened to nectar consistency, and he eventually advanced his abilities to be able to drink from a straw and open cup. Client \#3 had extensive stimulation to improve his oral motor quality and decrease the speed of eating. He became adept at eating at a slower pace and appeared to be chewing his foods better prior to swallowing. Mixed textured foods such as a fruit cup in juice or cereal with milk were tried to work on segmenting the bolus and differentiate the swallowing mechanism. Client \#3 had a repeated MBSS conducted, and he demonstrated marked improvement compared to previous studies and safe swallowing on all thicknesses and textures.

Client \#4 had an initial MBSS conducted where she demonstrated aspiration of thin liquids with inconsistent protective cough. Following this study, Client \#4 had 63 total sessions NMES two times per week with electrode placement just above the thyroid notch (see Picture 2 - placement 1/Pediatric Placement A). In addition to NMES, all liquids were thickened to nectar consistency. She had a rapid oral transit mechanism, and would often stuff her mouth while eating. Client \#4 also had excessive drooling with the need for 5-6 heavy terry cloth bibs per day. Client \#4 had extensive oral awareness therapy conducted to improve sensitivity in her mouth and on her tongue using vibration with z-vibe and textured brushes. Client \#4 also completed simple exercises to improve her tongue strength and dexterity during the course of intervention. Tongue lateralization and extension were practiced with and without a mirror. This client had 39 sessions of NMES, then Client \#4 had extensive back surgery completed during the course of therapy intervention to correct a spinal curvature and remove an extra hemi vertebrae. She was hospitalized for 11 days with a break in dysphagia therapy services and a break from NMES. Subsequently, Client \#4 had an issue with her internal spinal fixation and required a repeated fixation surgery. Her hospitalization this time was 7 days, and her external fixation was a brace that encompassed her entire spine including her jaw. The brace was attached to her head and prevented her from moving her neck. She was unable to receive NMES for 110 days, however, the same precautions were continued (thickened liquids to 
nectar consistency and pacing of solids while eating). NMES resumed (an additional 24 sessions) following brace removal; her repeat MBSS demonstrated oral and pharyngeal phases were within normal limits. There was no aspiration identified during the exam.

Client \#5 had her initial MBSS conducted; she demonstrated aspiration with thin liquid, not seen on radiographic images but captured after the child coughed when the radiologist turned the x-ray back on to check if her lungs were clear. Evaluation with nectar thick liquid by bottle demonstrated multiple normal swallows; however, towards the end of the study, the child tired and there was an episode of penetration and small aspiration with the child coughing. Following this study, Client \#5 had 26 sessions of NMES with electrode placement at the level of the thyroid notch (see Picture 2 - placement 2/Pediatric Placement B). She also had extensive therapy done to advance her food textures from smooth stage I foods like single ingredient introductory foods (e.g. pears, squash) to stage III (mixed textured foods that have noticeable chunks of foods mixed into a puree) foods and table foods. In addition, sippy cup drinking was introduced and the ability to self feed. Client \#5 had her MBSS repeated, and the study demonstrated minimal premature laryngeal penetration with no evidence of aspiration.

Table 2. Pre-/Post-NMES Findings as Per Radiologist MBSS Reports

\begin{tabular}{|c|c|c|c|}
\hline NAME & PRE-NMES FINDINGS & POST-NMES FINDINGS & $\begin{array}{l}\text { TIME INTERVAL } \\
\text { BETWEEN STUDIES }\end{array}$ \\
\hline Client \#1 & $\begin{array}{l}\text { Persistent, silent micro aspiration with } \\
\text { both rice cereal and thin barium by } \\
\text { bottle }\end{array}$ & $\begin{array}{l}\text { No aspiration or penetration with } \\
\text { rice cereal, thin barium by bottle or } \\
\text { any other consistency. }\end{array}$ & 8 months \\
\hline \multirow[t]{3}{*}{ Client \#2 } & $\begin{array}{l}\text { Oral phase mildly compromised with } \\
\text { more significant compromise of the } \\
\text { pharyngeal phase. }\end{array}$ & $\begin{array}{l}\text { Coordinated oral and pharyngeal } \\
\text { phase }\end{array}$ & 3 months \\
\hline & Silent aspiration on water consistency & $\begin{array}{l}\text { No aspiration or penetration with } \\
\text { water consistency }\end{array}$ & \\
\hline & Incomplete laryngeal closure & Complete laryngeal closure & \\
\hline Client \#3 & $\begin{array}{l}\text { Persistent silent aspiration on thin } \\
\text { liquids }\end{array}$ & $\begin{array}{l}\text { No aspiration on thin liquids. Safe } \\
\text { on all thin liquids in any format } \\
\text { (e.g. open cup, straw or sippy cup) }\end{array}$ & 3.5 months \\
\hline Client \#4 & $\begin{array}{l}\text { Aspiration on thin liquids, inconsistent } \\
\text { protective cough }\end{array}$ & $\begin{array}{l}\text { No aspiration on thin liquids. No } \\
\text { coughing with liquids during the } \\
\text { study. }\end{array}$ & 4 months \\
\hline Client \#5 & $\begin{array}{l}\text { Aspiration on thin liquids not seen on } \\
\text { radiographic images, but after client } \\
\text { coughed. Tiring towards end of study } \\
\text { with aspiration of nectar thick liquid }\end{array}$ & $\begin{array}{l}\text { No evidence of aspiration. Minimal } \\
\text { premature laryngeal penetration as } \\
\text { study progressed and client tired. } \\
\text { No coughing during the study. }\end{array}$ & 7 months \\
\hline
\end{tabular}

\section{DISCUSSION}

All five children improved their swallowing function after receiving a course of dysphagia therapy with NMES and oral stimulation, along with natural maturation. Each radiologist reported improved swallow safety and the ability to advance in consistencies. Swallowing was stronger and more prompt and each child was deemed "safe" on at least one consistency or texture as compared to pre-NMES studies. Each child had a report completed by the attending radiologist with the radiologist reports used to determine the study outcomes and to document change. In the case of the five children outlined in this case series, NMES played a role in improving swallowing function and overall quality of life. This case series could suggest that NMES is an effective intervention for a child diagnosed with pharyngeal phase dysphagia.

Limitations of this study include varied lengths of time for each child's treatment. A variety of diagnoses made it difficult to compare outcomes, and limitations of using the radiologist report as a benchmark for change should be considered when 
weighing the results of this study. Depending on the particular circumstances of each child, NMES was provided over a longer period of time, thus maturation is a factor that could account for swallow improvement. This case series is limited to just five children who had both an initial MBSS and discharge MBSS. Other children were not included who did not meet the beginning/ending MBSS criteria. However, despite the limitations of the case series design, this author predicts that the information gained can add to the body of literature on the effectiveness of NMES in the pediatric population. Additional research needs to be conducted to further test the efficacy of NMES with larger sample sizes and age ranges especially as it relates to home-based early intervention.

\section{REFERENCES}

1. Pennsylvania Office of Child Development and Early Learning. Pennsylvania's Approach to the Delivery of Early Intervention Services. 2008.

2. Pennsylvania Department of Education and Department of Public Welfare. Infants-Toddlers Pennsylvania Learning Standards for Early Childhood. 2009.

3. Logemann JA. Manual for the Videofluorographic Study of Swallowing. Second Edition, Austin, TX 1993.

4. Wijting Yo, Freed M. Vital Stim $®$ Certification Program: Training Manual for the use of Neuromuscular Electrical Stimulation in the Treatment of Dysphagia. CIAO Seminars, Pensacola, FL 2006.

5. Vital Stim® User Manual. Chattanooga Group, Encore Medical Corporation, Austin, TX. 2005.

6. Freed M. Use of electric stimulation to restore swallow function. FDA trial data; 1998.

7. Blumenfeld L, Hahn Y, Lepage A, Leonard R, Belafsky PC. Transcutaneous electrical stimulation versus traditional dysphagia therapy: A nonconcurrent cohort study. Otolaryngol Head Neck Surg. Nov 2006;135(5):754-7. [PMID 17071307]

8. Carnaby-Mann G, Crary M. Neuromuscular electrical stimulation for treatment of refractory dysphagia: A case series report. Gainsville, FL:University of Florida; 2007.

9. Christiaanse M, Glynn J, Bradshaw J. Experience with transcutaneous electrical stimulation: A new treatment option for the management of pediatric dysphagia. Conference Proceeding: NCSHA. Charleston; 2003.

10. FDA. VitalStim 510 (k) clearance document K023347. 2002

11. Huckabee ML, Doeltgen S. Emerging modalities in dysphagia rehabilitation: neuromuscular electrical stimulation. The New Zealand Medical Journal. 12 October 2007;120(1263);U2744. [PMID 17972964]

12. Xia W, Zheng C, Lei $Q$, Tang Z, Hua Q, Zhang Y, Zhu S. Treatment of post-stroke dysphagia by vitalstim therapy coupled with conventional swallowing training. J Huazhong Univ Sci Technolog Med Sci. 2011 Feb;31(1):73-6. [PMID 21336727]

13. Ryu JS, Kang JY, Park JY, Nam SY, Choi Sh, Roh JL, Kim SY, Choi KH. The effect of electrical stimulation therapy on dysphagia following treatment for head and neck cancer. Oral Oncology. 2009 Aug;45(8):665-8. Doi: 10.1016/j.oraloncology, 2008. 10.005.

14. Oh KY, Kim SA, Kwon KH, Kim SW, Lee SY. The effects of neuromuscular electrical stimulation on swallowing function in healthy older adults. J Korean Acad Rehabil Med. 2011;35(2):195-200. Accessed June 20, 2012. http://koreamed.org/

15. Langdon C, Blacker D. Dysphagia in stroke: a new solution. SAGE-Hindawi Access to Research Stroke Research and Treatment. 2010, Article ID 570403, 6 pages. doi:10.4061/2010/570403 Accessed June 20, 2012. downloads.hindawi.com/journals/srt/2010/570403.pdf

16. Barikroo A, Lam PM. Comparing the effects of rehabilitation swallowing therapy vs. functional neuromuscular electrical stimulation therapy in an encephalitis patient: a case study. Dysphagia. 2011 Dec;26(4):418-23. [PMID 21267746]

\section{KEY TERMS}

Neuromuscular Electrical Stimulation (NMES), Modified Barium Swallow Study (MBSS), Early Intervention (EI), Dysphagia, VitalStim ${ }^{\circledR}$ 